\title{
MEASURE SPLITTING AND AVERAGE MEASURABILITY
}

\author{
TREVOR J. MCMINN
}

1. Introduction. Theorem 3.8 below is a reduction theorem for measure splitting defined in 3.1. A theorem preliminary to it is applied in the proof of 4.1 , but, we feel, 3.8 is of some interest in itself.

Theorems 4.3 and 4.8 , based on the known fact that the average of the inner and outer measures of a measure is a measure, give necessary and sufficient conditions ${ }^{1}$ for every average-measurable set to be outer-measurable. These conditions are framed in terms of splitting and straddling, 4.5.

2. Preliminary definitions and theorems. We agree that $\omega$ is the set of non-negative integers and that each such integer is the set of all such less than it.

By $N(F)$ we mean the number of members of $F$ if $F$ is finite and $\infty$ otherwise.

By sng $x$ we mean the set whose sole member is $x$.

By $\sigma F$ we mean the union of members of $F$.

By $\sim A$ we mean the set of points not in $A$.

2.1. Definition. $m$ measures $S$ if and only if $m$ is such a function on

$$
\mathrm{E} \beta(\beta \subset S) \text { to } \mathrm{E} t(0 \leqq t \leqq \infty)
$$

that

$$
m(A) \leqq \sum_{\alpha \in H} m(\alpha),
$$

whenever $H$ is such a countable family that $A \subset \sigma H \subset S$.

2.2. Definition. $A$ is $m$ measurable if and only if $A \in$ domain $m$ and, for each $T \in$ domain $m$,

$$
m(T)=m(T A)+m(T \sim A) .
$$

By $\mathrm{mbl} m$ we mean the family of $m$ measurable sets.

2.3. Definitions. We define functions im $m$, om $m$, and av $m$ upon domain $m$ such that, for $A \in$ domain $m$,

$.1 \mathrm{im} m(A)=\sup _{\alpha \in H} m(\alpha)$, where $H=(\operatorname{mbl} m) \cap \mathrm{E} \beta(\beta \subset A)$,

.2 om $m(A)=\inf \alpha_{\alpha \in G} m(\alpha)$, where $G=(\operatorname{mbl} m) \cap E \beta(A \subset \beta)$,

.3 av $m(A)=(\operatorname{im} m(A)$ tom $m(A)) / 2$.

2.4. Definitions.

Received by the editors October 12, 1953 and, in revised form, November 19, 1953.

1 The present paper is an outgrowth of questions concerning such conditions raised by A. P. Morse which he himself partially answered in the theorem mentioned herein following the proof of 4.8 . 
.1 kernel $(m, A)=\mathrm{E} A_{*}\left(A \supset A_{*} \in \mathrm{mbl} m\right.$ and $\left.\operatorname{im} m(A)=m\left(A_{*}\right)\right)$,

.2 hull $(m, A)=\mathrm{E} A^{*}\left(A \subset A^{*} \in \mathrm{mbl} m\right.$ and om $\left.m(A)=m\left(A^{*}\right)\right)$.

We henceforth assume that $\mathcal{S}$ is a fixed set and that $\phi$ measures $\mathcal{S}$. The theorems in this section are known. For proofs of 2.5 through 2.12 , as well as other elementary measure theoretic results we use, see [1]. For proofs of $2.13,2.14$, and 2.15 see [2]. 2.16 is a slight generalization of a similar theorem in [1]. 2.17.1-2.17.4 are easily checked.

2.5 through 2.15 are referred to in the sequel. $2.16,2.17$, and 2.18 invite comparison with 4.3 and 4.8 .

2.15 emphasizes the great difference in the Carathéodory definition of measurability, 2.2, and one which might be based upon additivity of a set and its complement or, for Lebesgue measure, additivity, for each interval, of the intersection of the set with the interval and its complement with respect to the interval. Under such a definition, every set would be average-measurable.

2.5. Theorem. If $H$ is disjointed, $H \subset \mathrm{mbl} \phi, \phi(\sigma H)<\infty$, and 0 $<\phi(\beta)$, whenever $\beta \in H$, then $H$ is countable.

\subsection{TheOREMS.}

.1 domain $\phi=$ domain im $\phi=$ domain om $\phi=$ domain av $\phi$,

$.2 A \subset S$ implies im $\phi(A) \leqq \phi(A) \leqq$ om $\phi(A)$,

.3 $A \in \mathrm{mbl} \phi$ implies $\phi(A)=\operatorname{im} \phi(A)=$ om $\phi(A)=\operatorname{av} \phi(A)$.

2.7. TheOREM. om $\phi$ measures $\mathcal{S}$.

2.8. Theorem. If $A \subset \mathcal{S}$, then hull $(\phi, A) \neq 0$ and kernel $(\phi, A) \neq 0$.

2.9. Theorem. If $A \subset \mathcal{S}, A_{*} \in$ kernel $(\phi, A), A^{*} \in$ hull $(\phi, A)$, $\phi\left(A^{*}\right)<\infty, B \in \mathrm{mbl} \phi$, then $A_{*} B \in$ kernel $(\phi, A B)$ and $A^{*} B \in$ hull $(\phi, A B)$.

2.10. Theorem. If $A \cup B \in \mathrm{mbl} \phi$ and $A B=0$, then im $\phi(A)$ tom $\phi(B)=\phi(A \cup B)$.

2.11. Theorem. $\mathrm{mbl} \phi \subset \mathrm{mbl}$ om $\phi$.

2.12. Theorem. If $H$ is countable, $H$ is disjointed, and $H \subset \mathrm{mbl} \phi$, then, for each $T \subset S$,

$$
\operatorname{im} \phi(T \sigma H)=\sum_{\beta \in H} \operatorname{im} \phi(T \beta) .
$$

2.13. ThEOREM. av $\phi$ measures $S$.

2.14. Theorem. $\mathrm{mbl}$ om $\phi \subset \mathrm{mbl}$ av $\phi$.

2.15. Theorem. If $A \subset \mathcal{S}$ and $T \in \mathrm{mbl}$ om $\phi$, then 


$$
\operatorname{av} \phi(T)=\operatorname{av} \phi(T A)+\operatorname{av} \phi(T \sim A) .
$$

2.16. Theorem. If $A \in \mathrm{mbl}$ om $\phi$ and, for $T \subset \mathcal{S}, \phi(T A)<\infty \mathrm{im}$ plies om $\phi(T A)<\infty$, then $A \in \mathrm{mbl} \phi$.

2.17. TheOREMS.

.1 om om $\phi=$ om $\phi$,

.2 av om $\phi=a v \phi$,

$.3 \mathrm{mbl}$ av $\phi \subset \mathrm{mbl}$ om $\phi$ implies om av $\phi=0 \mathrm{~m} \phi$,

$.4 \mathrm{mbl}$ av $\phi \subset \mathrm{mbl}$ om $\phi$ implies av av $\phi=$ av $\phi$.

2.18. Remarks. The following is not a theorem: If $\mathrm{mbl}$ om $\phi$ $\subset \mathrm{mbl} \phi$, then, for $T \subset \mathcal{S}, \phi(T)<\infty$ implies om $\phi(T)<\infty$.

Converses of 2.17 .3 and 2.17 .4 are not theorems.

\section{Measure splitting.}

3.1. Definition. Fn splits if and only if

(i) $n \in \omega$,

(ii) $F$ is nonvacuous, countable, and disjointed,

(iii) $\sigma F \in \mathrm{mbl} \phi$,

(iv) $\beta \in F$ implies $0<\phi(\beta)$,

(v) $\operatorname{im} \phi(\sigma G)=0$, whenever $G \subset F$ and $N(G) \leqq n$.

3.2. Remarks. If $\sigma F \in \mathrm{mbl} \phi$ and $\phi(\sigma F)<\infty$, then by the use of 2.10 it can be shown that (i) and (ii) below are equivalent:

(i) $\operatorname{im} \phi(\sigma F \sim \beta)=0$, for $\beta \in F$;

(ii) om $\phi(\beta)=\phi(\sigma F)$, for $\beta \in F$.

It is easy to find a measure and a family which $n$ splits, for each $n \in \omega$, but for which neither (i) nor (ii) holds. However, if $F$ is infinite, then (i) obviously implies $3.1(\mathrm{v})$, for each $n \in \omega$.

If $\mathcal{S}$ is a complete separable metric space, $T \in \mathrm{mbl} \phi, 0<\phi(T)<\infty$, $\phi(A \cup B)=\phi(A)+\phi(B)$, whenever $A$ and $B$ are a positive distance apart, and every subset of $\mathcal{S}$ is contained in a Borel set of the same $\phi$ measure (for instance Lebesgue measure on the reals), then, by the usual method of transfinite induction on the perfect subsets, a disjointed family $F$, whose union is $T$, with preassigned finite or countable infinite number of members can be constructed satisfying (i).

Our objective in this section is to establish the reduction Theorem 3.8. However, the heart of the problem is embodied in the proofs of 3.5 and 3.7 .

3.3. THEOREM. If

(i) $U_{F^{\prime} \in K}$ sng $\sigma F^{\prime}$ is nonempty, countable, and disjointed,

(ii) $\phi\left(M \sim \bigcup_{F^{\prime} \in K} \sigma F^{\prime}\right)=0$,

(iii) $d \in \omega$ and $n \in \omega$, 
(iv) $N\left(F^{\prime}\right)=d$ and $F^{\prime} n \phi$ splits, for $F^{\prime} \in K$, then there is a $G$ such that $N(G)=d, \sigma G=M$, and $G n \phi$ splits.

Proof. Let $\eta$ be a function on $K$ such that $\eta\left(F^{\prime}\right)$ is a $d$-term sequence of members of $F^{\prime}$, for $F^{\prime} \in K$. Let

$$
G^{\prime}=\bigcup_{i \in{ }_{d}} \operatorname{sng} \bigcup_{F^{\prime}} \in \in_{K} \eta\left(F^{\prime}\right)_{i} .
$$

Clearly $N\left(G^{\prime}\right)=d$ and $\sigma G^{\prime}=\bigcup_{F^{\prime} \in K} \sigma F^{\prime}$.

To show that $G^{\prime} n \phi$ splits, we take an $n$-term univalent sequence $p$ such that $p_{i} \in d$, for $i \in n$. We then let

$$
G^{\prime \prime}=\bigcup_{i \in n} \text { sng } \bigcup_{F^{\prime}} \in K \eta\left(F^{\prime}\right)_{p_{i}} \text {. }
$$

Now since $F^{\prime} n \phi$ splits and $\bigcup_{i \in n} \eta\left(F^{\prime}\right)_{p_{i}} \subset \sigma F^{\prime}$, for $F^{\prime} \in K$, we apply 2.12 to get

$$
\begin{aligned}
\operatorname{im} \phi\left(\sigma G^{\prime \prime}\right) & =\operatorname{im} \phi\left(\bigcup_{i \in{ }_{n}} \bigcup_{F^{\prime} \in K} \eta\left(F^{\prime}\right)_{p_{i}}\right) \\
& =\operatorname{im} \phi\left(\bigcup_{F^{\prime} \in \in_{K}} \bigcup_{i \in n} \eta\left(F^{\prime}\right)_{p_{i}}\right) \\
& =\sum_{F^{\prime} \in K} \operatorname{im} \phi\left(\bigcup_{i \in n} \eta\left(F^{\prime}\right)_{p_{i}}\right)=0 .
\end{aligned}
$$

Thus $G^{\prime} n \phi$ splits.

By joining $M \sim \bigcup_{F^{\prime} \in K} \sigma F^{\prime}$ to one of the members of $G^{\prime}$ we get such a set $G$ that $N(G)=d, \sigma G=M$, and $G n \phi$ splits.

3.4. Theorem. If $F n \phi$ splits, $H \subset F, N(H) \leqq n, A \subset \sigma F, A \in \mathrm{mbl} \phi$, and $0<\phi(A)$, then for some $\alpha \in F \sim H, 0<\operatorname{om} \phi(A \alpha)$.

Proof. We use 2.10, 3.1 (v) and 3.1 (ii) to get

$$
\begin{aligned}
0<\phi(A)=\phi(A \sigma F) & =\text { om } \phi(A \sigma F \sim \sigma H)+\operatorname{im} \phi(A \sigma H) \\
& =\text { om } \phi(A \sigma(F \sim H)) \\
& \leqq \sum \alpha \in(\mathbb{F} \backsim A) \text { om } \phi(A \alpha) .
\end{aligned}
$$

3.5. TheOREM. If $F n \phi$ splits and $\phi(\sigma F)<\infty$, then there are sets $K$ and $A$ for which

(i) $K \subset F$ and $N(K)=n+1$,

(ii) $A \subset \sigma F, A \in \mathrm{mbl} \phi$, and $0<\phi(A)$,

(iii) om $\phi(A \alpha)=\phi(A)$, for each $\alpha \in K$.

Proof. ${ }^{2}$ For $K \subset F$ let $\mu(K)$ be the family consisting of those sets $A$ for which $A \subset \sigma F, A \in \mathrm{mbl} \phi, 0<\phi(A)$, and om $\phi(A \alpha)=\phi(A)$, for each $\alpha \in K$. Let $\Delta$ be the collection of those families $K$ for which $K \subset F, N(K) \leqq n+1$, and $\mu(K) \neq 0$.

2 The proof included here is due to D. D'Esopo and improves on the author's by avoiding the principle of choice and an inductive definition. 
If $\sup { }_{K \in \Delta} N(K)=n+1$, then the theorem is proved.

If otherwise, then, since $0 \in \Delta$, we have $0 \neq \Delta$ and can choose $H \in \Delta$ for which $N(H)=\sup _{K \in \Delta} N(K) \leqq n$. Since $\mu(H) \neq 0$, we let $A \in \mu(H)$ and use 3.4 to select $\alpha \in F \sim H$ so that $0<$ om $\phi(A \alpha)$. Then choose $A^{\prime} \in$ hull $(\phi, A \alpha)$ so that $A^{\prime} \subset A$. Finally let $K^{\prime}=H \cup_{\text {sng }} \alpha$. Noting that $A^{\prime} \alpha=A \alpha$ we have

$$
\phi\left(A^{\prime}\right)=\text { om } \phi(A \alpha)=\text { om } \phi\left(A^{\prime} \alpha\right),
$$

while for $\gamma \in H$, using 2.10 twice, the fact that $\phi(A) \leqq \phi(\sigma F)<\infty$, and the fact that $A \in \mu(H)$, we have

$$
\begin{aligned}
\phi\left(A^{\prime}\right) & =\text { om } \phi\left(A^{\prime} \gamma\right)+\operatorname{im} \phi\left(A^{\prime} \sim \gamma\right) \\
& \leqq \text { om } \phi\left(A^{\prime} \gamma\right)+\operatorname{im} \phi(A \sim \gamma) \\
& =\text { om } \phi\left(A^{\prime} \gamma\right)+\phi(A)-\text { om } \phi(A \gamma) \\
& =\text { om } \phi\left(A^{\prime} \gamma\right) \leqq \phi\left(A^{\prime}\right) .
\end{aligned}
$$

Hence $A^{\prime} \in \mu\left(K^{\prime}\right), K^{\prime} \in \Delta$, and

$$
N\left(K^{\prime}\right) \leqq \sup _{K \in \Delta} N(K)=N(H)<N\left(K^{\prime}\right) .
$$

Contradiction. Hence the theorem.

3.6. Theorem. If $F n \phi$ splits and $\phi(\sigma F)<\infty$, then there is such an $F^{\prime}$ that $N\left(F^{\prime}\right)=n+1, \sigma F^{\prime} \subset \sigma F$, and $F^{\prime} n \phi$ splits.

Proof. Choose $K$ and $A$ according to 3.5. Let $F^{\prime \prime}=\bigcup_{\alpha \in K}$ sng $(A \alpha)$ and let $F^{\prime}$ be a family obtained by joining $A \sim \sigma F^{\prime \prime}$ to one of the members of $F^{\prime \prime}$. Clearly $\sigma F^{\prime}=A \subset \sigma F$ and $A \in \mathrm{mbl} \phi$. Also $0<\phi(A)$ =om $\phi(\beta)$, for $\beta \in F^{\prime}$, hence $N\left(F^{\prime}\right)=n+1$ and $0<\phi(\beta)$, for $\beta \in F^{\prime}$. Since $\phi(A)<\infty$, we use 2.10 to infer that $0=\phi(A)-$ om $\phi(\beta)$ $=\operatorname{im} \phi(A \sim \beta)$, for $\beta \in F^{\prime}$. We conclude that $F^{\prime} n \phi$ splits.

3.7. Theorem. If $F n \phi$ splits and $\phi(\sigma F)<\infty$, then there is such $a G$ that $N(G)=n+1, \sigma G=\sigma F$, and $G n \phi$ splits.

Proof. Let $G$ be the collection of all families $K$ such that

$\bigcup_{F^{\prime}} \in \in_{K} \operatorname{sng} \sigma F^{\prime}$ is disjointed,

and, for $F^{\prime} \in K$,

$$
N\left(F^{\prime}\right)=n+1, \sigma F^{\prime} \subset \sigma F \text {, and } F^{\prime} n \phi \text { splits. }
$$

We resort to Zorn's Lemma to extract from $G$ a nest $\mathcal{N}$ such that no member of $G \sim \mathcal{N}$ contains $\sigma \mathcal{N}$. Let $H=\bigcup_{F^{\prime} \in \sigma \mathcal{N}} \operatorname{sng} \sigma F^{\prime}$. Clearly $H$ is disjointed. By $2.5, H$ is countable. Hence $\sigma H \in \mathrm{mbl} \phi$.

Let 


$$
F^{\prime \prime}=\mathrm{E} \beta \text { (for some } \alpha \in F, \beta=\alpha \sim \sigma H \text { and } 0<\phi(\beta) \text { ). }
$$

Now $\sigma F^{\prime \prime}=\sigma F \sim \sigma H \in \mathrm{mbl} \phi$. Hence $F^{\prime \prime} \neq 0$ implies $F^{\prime \prime} n \phi$ splits. Since $\sigma \mathcal{N}$ is maximal, we use 3.6 to see that $F^{\prime \prime}=0$. But, since $F$ is countable, $F^{\prime \prime}=0$ implies $\phi(\sigma F \sim \sigma H)=0$. Hence by 3.3 there is such a $G$ that $N(G)=n+1, \sigma G=\sigma F$, and $G n \phi$ splits.

3.8. Theorem. If $F n \phi$ splits, $\phi(\sigma F)<\infty, k \in \omega$, and $n<k \leqq N(F)$, then there is such an $F^{\prime}$ that $N\left(F^{\prime}\right)=k, \sigma F^{\prime}=\sigma F$, and $F^{\prime} n \phi$ splits.

Proof. Use 3.7 to secure such a $G$ that $N(G)=n+1, \sigma G=\sigma F$, and $G n \phi$ splits. Let $H=\bigcup_{\alpha \in G}$ sng $\bigcup_{\beta \in F}$ sng $(\alpha \beta)$. Let $p$ be such a function on $H$ that $p(a) \in a$ and $0<\phi(p(a))$, for $a \in H$. Choose a family $H^{\prime}$ consisting of $k-(n+1)$ members of $\sigma H \sim \bigcup_{a \in H}$ sng $p(a)$ each of positive $\phi$ measure. Let

$$
F^{\prime}=H^{\prime} \cup \bigcup_{a} \in H \text { sng }\left(p(a) \cup\left(\sigma a \sim \sigma H^{\prime}\right)\right) .
$$

Check that $N\left(F^{\prime}\right)=N\left(H^{\prime}\right)+N(G)=k-(n+1)+n+1=k, F^{\prime}$ is disjointed, $\sigma F^{\prime}=\sigma F$, and, since $G n \phi$ splits, that $F^{\prime} n \phi$ splits.

3.9. Remark. Examples can be constructed where it is impossible to choose $G$ according to 3.7 so that $F$ is a refinement of $G$, that is, so that every member of $F$ is contained in some member of $G$. Thus $G$ cannot, in general, be obtained merely by joining together members of $F$.

\section{Average measurability.}

4.1. Theorem. If (sng $A \cup$ sng $B) 1 \phi$ splits, $\phi(A \cup B)<\infty$, and $A \notin \mathrm{mbl}$ av $\phi$, then there is such an $F^{\prime}$ that $N\left(F^{\prime}\right)=3, \sigma F^{\prime} \subset A \cup B$, and $F^{\prime} 2 \phi$ splits.

Proof. Let $Z$ be such that

$$
\operatorname{av} \phi(Z)<\operatorname{av} \phi(Z A)+\operatorname{av} \phi(Z \sim A) .
$$

Furthermore let

$$
\begin{aligned}
Z^{*} & \in \text { hull }(\phi, Z), \quad Z_{*} \in \text { kernel }(\phi, Z), \\
X & =(A \cup B) Z^{*} \sim Z_{*}, \\
W & =X Z, \quad V=X \sim Z, \\
P & \in \text { kernel }(\phi, W A \cup V B), \quad Q \in \operatorname{kernel}(\phi, W B \cup V A), \\
C & =X \sim P \sim Q, \\
F & =\operatorname{sng}(C W A) \cup \operatorname{sng}(C W B) \cup \operatorname{sng}(C V A) \cup \operatorname{sng}(C V B) .
\end{aligned}
$$

We complete the proof in four parts. 
PART I. om $\phi(W)<$ om $\phi(W A)$ tom $\phi(W B)$.

Proof. Since $A \cup B \in \mathrm{mbl}$ av $\phi, 2.11$ and 2.14 , we test with $Z \sim A$ to get

$$
\text { av } \phi(Z \sim A)=\operatorname{av} \phi(Z B)+\operatorname{av} \phi(Z \sim(A \cup B)),
$$

and test with $Z$ and use (1) and (2) to get

$$
\begin{aligned}
& \operatorname{av} \phi(Z(A \cup B))+\operatorname{av} \phi(Z \sim(A \cup B)) \\
& \quad=\operatorname{av} \phi(Z)<\operatorname{av} \phi(Z A)+\operatorname{av} \phi(Z B)+\operatorname{av} \phi(Z \sim(A \cup B)) .
\end{aligned}
$$

Since (1) assures us that av $\phi(Z)<\infty$, subtraction in (3) leads to

$$
\text { av } \phi(Z(A \cup B))<\operatorname{av} \phi(Z A)+\operatorname{av} \phi(Z B) \text {. }
$$

We use the fact that $Z_{*} \in \mathrm{mbl}$ av $\phi,(4)$, again $Z_{*} \in \mathrm{mbl}$ av $\phi, 2.15$, and subtraction to get

$$
\begin{aligned}
\operatorname{av} \phi\left(Z(A \cup B) Z_{*}\right)+\operatorname{av} \phi\left(Z(A \cup B) \sim Z_{*}\right) \\
=\operatorname{av} \phi(Z(A \cup B))<\operatorname{av} \phi(Z A)+\operatorname{av} \phi(Z B) \\
=\operatorname{av} \phi\left(Z A Z_{*}\right)+\operatorname{av} \phi\left(Z A \sim Z_{*}\right)+\operatorname{av} \phi\left(Z B Z_{*}\right)+\operatorname{av} \phi\left(Z B \sim Z_{*}\right) \\
=\operatorname{av} \phi\left(Z(A \cup B) Z_{*}\right)+\operatorname{av} \phi\left(Z A \sim Z_{*}\right)+\operatorname{av} \phi\left(Z B \sim Z_{*}\right), \\
\quad \operatorname{av} \phi(W)<\operatorname{av} \phi(W A)+\operatorname{av} \phi(W B) .
\end{aligned}
$$

Since clearly im $\phi(W)=0$, we have

$$
\text { om } \phi(W)<\text { om } \phi(W A)+\text { om } \phi(W B) .
$$

PART II. $0<\phi(\sigma F)$.

Proof. Suppose $\phi(C)=0$. Then using 2.9, 2.11, and 2.6.3, we get

$$
\begin{aligned}
\text { om } \phi(W) & =\phi(X)=\phi(C)+\phi(P)+\phi(Q), \\
\text { om } \phi(W A) & =\text { om } \phi(X W A)=\text { om } \phi(C W A \cup P W A) \\
& =\text { om } \phi(C W A)+\text { om } \phi(P W A) \\
& \leqq \text { om } \phi(P)=\phi(P), \\
\text { om } \phi(W B) & \leqq \text { om } \phi(Q)=\phi(Q) .
\end{aligned}
$$

Now referring to Part I we get

$$
\text { om } \begin{aligned}
\phi(W) & <\text { om } \phi(W A)+\text { om } \phi(W B) \\
& \leqq \phi(P)+\phi(Q)=\text { om } \phi(W) .
\end{aligned}
$$

Contradiction. Hence $0<\phi(C)$. Since $\sigma F=C(A \cup B)(W \cup V)=C$, we have $0<\phi(\sigma F)$.

PART III. $\alpha \in F$ and $\beta \in F$ imply im $\phi(\alpha \cup \beta)=0$. 
Proof. We observe that im $\phi(W)=\operatorname{im} \phi(V)=0$. Now if $\alpha \in F$ and $\beta \in F$, then

$$
\begin{gathered}
\alpha \cup \beta \subset A \text { or } \alpha \cup \beta \subset B \text { or } \alpha \cup \beta \subset W \text { or } \alpha \cup \beta \subset V \text { or } \\
\alpha \cup \beta=C(W A \cup V B) \text { or } \alpha \cup \beta=C(W B \cup V A) .
\end{gathered}
$$

But since $C P=C Q=0$, we have, using 2.9 , im $\phi(\alpha \cup \beta)=0$.

PART IV. For some $F^{\prime}, N\left(F^{\prime}\right)=3, \sigma F^{\prime} \subset \sigma F$, and $F^{\prime} 2 \phi$ splits.

Proof. ${ }^{3}$ If for some $\beta \in F, \phi(\beta)=0$, then the existence of $F^{\prime}$ is obvious in view of Part II and Part III.

If $\beta \in F$ implies $0<\phi(\beta)$, then by Part III $F 2 \phi$ splits. We now use 3.6.

The proof of the theorem is complete.

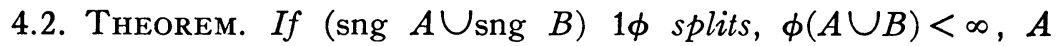
$\notin \mathrm{mbl}$ av $\phi$, and $\mathrm{im}$ av $\phi(A)=0$, then there is such a $G$ that $N(G)=3$, $\sigma G=A \cup B$, and $G 2 \phi$ splits.

Proof. As in the proof of 3.7, we use Zorn's Lemma and 4.1 to secure such a maximal family $\sigma \mathcal{N}$ that $\bigcup_{F^{\prime} \in \sigma \mathcal{N}}$ sng $F^{\prime}$ is disjointed, and, for $F^{\prime} \in \sigma \mathcal{N}, N\left(F^{\prime}\right)=3, \sigma F^{\prime} \subset A \cup B$, and $F^{\prime} 2 \phi$ splits. Let $H$ $=\bigcup_{F^{\prime} \in \sigma \mathcal{N}}$ sng $\sigma F^{\prime}$. By $2.5, H$ is countable. Clearly $0<\phi((A \cup B)$ $\sim \sigma H)$ implies (sng $(A \sim \sigma H) \cup$ sng $(B \sim \sigma H)) 1 \phi$ splits. Since $\sigma \mathcal{N}$ is maximal, $A \sim \sigma H \in \mathrm{mbl}$ av $\phi$ or $\phi((A \cup B) \sim \sigma H)=0$. But since im av $\phi(A)=0$, we have $A \sim \sigma H \in \mathrm{mbl}$ av $\phi$ implies av $\phi(A \sim \sigma H)=0$ which implies $\phi(A \sim \sigma H)=0$. But $\phi(A \sim \sigma H)=0$ implies $\phi(B \sim \sigma H)$ $=0$. Hence $\phi((A \cup B) \sim \sigma H)=0$. By 3.3 there is such a $G$ that $N(G)$ $=3, \sigma G=A \cup B$, and $G 2 \phi$ splits.

4.3. Theorem. If $\mathrm{mbl}$ av $\phi C \mathrm{mbl}$ om $\phi$, (sng $A \cup$ sng $B$ ) $1 \phi$ splits, and $\phi(A \cup B)<\infty$, then there is such $a G$ that $N(G)=3, \sigma G=A \cup B$, and $G 2 \phi$ splits.

Proof. Use 4.2.

4.4. Remark. That the expression obtained from 4.2 by deleting "im av $\phi(A)=0$ " is not a theorem can be shown with a five-element counterexample.

We have in 4.3 established a necessary condition that outer-measurable and average-measurable sets be the same. We proceed to the question of sufficiency and get an answer in 4.8 .

4.5. Definition. $G \phi$ straddles $F$ if and only if

(i) $G$ is countable, 3.6.

${ }^{3} \mathrm{By}$ means of other particular devices this part can be proved without the aid of 
(ii) $\sigma F \subset \sigma G$,

(iii) $A \in F, B \in F, \alpha \in G, \beta \in G$, and om $\phi(A \alpha)$ +om $\phi(B \beta)<\infty$ imply $\operatorname{im} \phi(A \alpha \cup B \beta)=0$.

4.6. Theorem. If $F 1 \phi$ splits, $\phi(\sigma F)<\infty$, and $G \phi$ straddles $F$, then $A \in F$ implies $A \notin \mathrm{mbl}$ av $\phi$.

Proof. Let $A \in F$. Choose $\alpha \in G$ so that $0<\phi(A \alpha)$ and choose $A^{\prime} \in$ hull $(\phi, A \alpha)$. Choose $B \in F$ so that $B \neq A$ and $0<\phi\left(A^{\prime} B\right)$. This is possible, for otherwise $\phi\left(A^{\prime} \sigma F \sim A\right)=0, A^{\prime} \sigma F \sim A \in \mathrm{mbl} \phi$, and $A \alpha$ $C A^{\prime} A \in \mathrm{mbl} \phi$ in contradiction to the hypothesis that $F 1 \phi$ splits. Choose $\beta \in G$ so that $0<\phi\left(A^{\prime} B \beta\right)$. Observe that

$$
\text { om } \phi(A \alpha)+\text { om } \phi(B \beta)+\phi\left(A^{\prime}\right) \leqq 3 \cdot \phi(\sigma F)<\infty .
$$

Now let $T=A^{\prime}(A \alpha \cup B \beta)$. Since $A \alpha \subset T \subset A^{\prime}$, we have

$$
\phi\left(A^{\prime}\right)=\text { om } \phi(A \alpha) \leqq \text { om } \phi(T) \leqq \text { om } \phi\left(A^{\prime}\right)=\phi\left(A^{\prime}\right)<\infty .
$$

But also we have om $\phi(T A)=$ om $\phi(A \alpha)$. Furthermore

$$
0<\phi\left(A^{\prime} B \beta\right) \leqq \text { om } \phi\left(A^{\prime} B \beta\right)=\text { om } \phi(T \sim A) .
$$

Consequently

$$
\text { om } \phi(T)<\text { om } \phi(T A)+\text { om } \phi(T \sim A) .
$$

We now use (5) and the fact that $G \phi$ straddles $F$ to decide that $\operatorname{im} \phi(T)=0$ and hence that

$$
\text { av } \phi(T)<\operatorname{av} \phi(T A)+\operatorname{av} \phi(T \sim A) .
$$

We conclude that $A \notin \mathrm{mbl}$ av $\phi$.

4.7. Theorem. If $D \in$ domain $\phi \sim \mathrm{mbl}$ om $\phi$, then for some $A$ and some $B, A \subset D, \phi(A \cup B)<\infty$, (sng $A \cup$ sng $B$ ) $1 \phi$ splits, and $A$ $\notin \mathrm{mbl}$ av $\phi$ implies $D \notin \mathrm{mbl}$ av $\phi$.

Proof. Since $D \in$ domain $\phi \sim \mathrm{mbl}$ om $\phi$, so choose $T$ that

$$
\text { om } \phi(T)<\text { om } \phi(T D)+\text { om } \phi(T \sim D)
$$

and let

$$
\begin{gathered}
T^{*} \in \text { hull }(\phi, T), \quad J \in \text { kernel }\left(\phi, T^{*} D\right), \quad A=T^{*} D \sim J, \\
A^{*} \in \text { hull }(\phi, A), \quad \text { and } \quad B=A^{*} \sim A .
\end{gathered}
$$

We have $A \subset D, A \cup B \in \mathrm{mbl} \phi$, and

$$
\phi(A \cup B)=\phi\left(A^{*}\right)=\text { om } \phi(A) \leqq \phi\left(T^{*}\right)=\text { om } \phi(T)<\infty .
$$

We assert that $0<\phi(A)$, for otherwise, $A \in \mathrm{mbl} \phi, T^{*} D \in \mathrm{mbl} \phi$, 
$T^{*} D \in \mathrm{mbl}$ om $\phi$, and hence

$$
\text { om } \begin{aligned}
\phi\left(T^{*}\right) & =\text { om } \phi\left(T^{*} D\right)+\text { om } \phi\left(T^{*} \sim\left(T^{*} D\right)\right) \\
& =\text { om } \phi\left(T^{*} D\right)+\text { om } \phi\left(T^{*} \sim D\right)
\end{aligned}
$$

in contradiction to the following inequality obtained from (6)

$$
\begin{aligned}
\operatorname{om} \phi\left(T^{*}\right)=\operatorname{om} \phi(T) & <\text { om } \phi(T D)+\text { om } \phi(T \sim D) \\
& \leqq \text { om } \phi\left(T^{*} D\right)+\text { om } \phi\left(T^{*} \sim D\right) .
\end{aligned}
$$

Since $\phi(A \cup B)<\infty$, it is easily checked that $\operatorname{im} \phi(A)+\operatorname{im} \phi(B)=0$

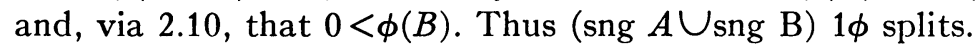

Since $\mathrm{mbl} \phi \subset \mathrm{mbl}$ av $\phi$, the conclusion follows from the definition of $A$.

4.8. Theorem. If for every $A$ and $B$ such that (sng $A \cup_{\text {sng } B)} 1 \phi$ splits and $\phi(A \cup B)<\infty$, there is such $a G$ that $G \phi$ straddles (sng $A$ $\cup$ sng $B)$, then $\mathrm{mbl}$ av $\phi \subset \mathrm{mbl}$ om $\phi$.

Proof. Use 4.6 and 4.7.

The conclusion of 4.8 is rather simply derived without the aid of 4.6 and 4.7 by a quite different method due to A. P. Morse from the following stronger hypotheses: For every $T \subset \mathcal{S}$ for which $0<\phi(T)<\infty$, there is a family $G$ such that $N(G)=\infty, T \subset \sigma G$, and $G n \phi$ splits for each $n \in \omega$.

Since $G 2 \phi$ splits and $\sigma G=A \cup B$ imply $G \phi$ straddles (sng $A \cup \operatorname{sng} B$ ) we may combine 4.3 and 4.8 to get the slightly less general but concise

4.9. Theorem. $\mathrm{mbl}$ av $\phi \subset \mathrm{mbl}$ om $\phi$ if and only if, for each $A$ and $B$ for which (sng $A \cup \operatorname{sng} B) 1 \phi$ splits and $\phi(A \cup B)<\infty$, there is a $G$ for which $N(G)=3, \sigma G=A \cup B$, and $G 2 \phi$ splits.

4.10. Theorem. If $\mathcal{S}$ is a complete separable metric space, $\phi(A \cup B)$ $=\phi(A)+\phi(B)$, whenever $A$ and $B$ are a positive distance apart, and every subset of $\mathcal{S}$ is contained in a Borel set of the same $\phi$ measure, then $\mathrm{mbl}$ av $\phi \subset \mathrm{mbl} \phi$.

Proof. In view of 3.2 , the hypothesis of 4.8 is satisfied. Since om $\phi$ $=\phi$, the conclusion of the theorem follows.

\section{REFERENCES}

1. H. Hahn and A. Rosenthal, Set functions, 1948, pp. 61-70.

2. C. Carathéodory, Vorlesungen über reelle Funktionen, Leipzig and Berlin, 1927, 2d ed., Note II, p. 693. 